Rev, Elev. Méd. vét. Pays trop., 1978, 31 (1) : 63-67.

\title{
Elimination des glossines et trypanosomose animale : Résultats de quelques sondages dans la région du lac Tchad
}

\author{
par Y. TAZE et J. GRUVEL \\ (avec la collaboration technique de Bouba BITSI)
}

\begin{abstract}
RESUMÉ
Les enquêtes par sondages effectuées dans la vallée du Bas-Chari au Tchad pour juger de l'incidence de la disparition des glossines et des traitements trypanocides de masse sur l'épidémiologic des trypanosomoses locales ( $T$. vivax et $T$. evansi) ont montré que 2 ans après la fin des pulvérisations la mortalité et la morbidité sévissant dans les troupeaux et attribuées par les éleveurs à ces hémo-parasites étaient uniquement provoquées par du parasitisme intestinal grave avec symptômes généraux pouvant prêter à confusion entre les diverses parasitoses.

Les auteurs concluent à l'efficacité des mesures appliquées dans l'élimination de trypanosomoses locales, et à la nécessité d'enquêtes épidémiologiques complètes, avant toute campagne d'éradication de glossines, tant pour fixer la véritable hiérarchie des maladies animales locales que pour définir celle des actions à entreprendre pour les combattre.
\end{abstract}

\section{CAMPAGNE DE LUTTE CONTRE LES GLOSSINES DANS LE BASSIN DU LAC TCHAD}

Une campagne de lutte contre Glossina tachinoides Westwood 1850 a été exécutée dans la vallée du bas-Chari, de N'Djamena jusqu'au lac Tchad, de 1971 à $1974(6,7)$.

Deux années consécutives de contrôle (1975 et 1976) ont montré l'absence de glossines dans toute la zone traitée $(2,7)$. Celle-ci fait partie du bassin du lac et englobe 2 cantons: Serbewel (au Cameroun) et Assalé (au Tchad), qui sont les 2 bénéficiaires des interventions d'assainissement.

L'élimination des tsé-tsé a profondément modifié le paysage de cette région. Dès 1975, des troupeaux de bovins et de petits ruminants ont envahi les zones riveraines où tout séjour prolongé leur était interdit auparavant. Les avantages de la lutte anti-glossines ont été vite connus des habitants et des éleveurs qui n'ont pas hésité à s'installer dès la fin des traitements et ont largement défriché la végétation des bords des cours d'eau.

La disparition des glossines constitue un pas décisif vers l'éradication des trypanosomoses animales, mais n'en est qu'une étape. Les autres diptères hématophages, difficilement contrôlables, contribuent à maintenir et à propager les trypanosomes au sein des troupeaux. Trypanosoma vivax peut persister par simple transmission mécanique hors des zones à glossines; $T$. evansi est transmis normalement de cette façon. Il n'est donc pas surprenant de rencontrer encore quelques foyers de maladie dans les régions assainies, entretenus par les animaux infestés localement ou récemment introduits. Les animaux malades et porteurs sains constituent en effet des réservoirs de parasites qui ne disparaîtront qu'avec le temps, à la suite de traitements trypanocides prolongés. 
De telles interventions ont été recommandées pendant au moins 2 ans, à la suite des pulvérisations contre les glossines.

\section{INFECTIONS TRYPANOSOMIENNES DANS LES RÉGIONS ASSAINIES}

Deux sondages ont été pratiqués, l'un en 1974 pendant les dernières pulvérisations contre les glossines, l'autre en 1977, 2 ans plus tard.

\section{SONDAGES DE 1974}

\section{a) Pratique des prélèvements}

- Ils ont été effectués, du 15 novembre au 15 décembre, dans quelques troupeaux non traités des cantons Serbewel et Assalé.

- Les trypanosomes ont été recherchés uniquement dans le sang des bovins malades ou très déficients ;

— par examen immédiat, à l'état frais, auprès de l'animal,

- par frottis de sang, colorés et examinés au laboratoire.

L'existence de quelques autres animaux infestés, parmi ceux non examinés n'est pas exclue car tous présentaient un état très médiocre.

\section{b) Résultats}

\section{1) Dans le canton Serbewel (Cameroun)}

Il n'a pas été possible de comparer le nombre d'animaux parasités à l'effectif total des troupeaux, qui est inconnu.

Sur 214 prélèvements répartis dans 10 localités du sultanat de Makari, 31 se sont révélés positifs à $T$. vivax, soit 14,43 p. 100 . Les pourcentages extrêmes relevés dans 2 villages sont de 25,90 et 8,30 p. 100 . Ces taux élevés peuvent s'expliquer par des infestations antérieures, contractées dans des zones à glossines, entretenues et dispersées ultérieurement - comme c'est la règle avant novembre en période de hautes eaux - par l'extrême abondance des Tabanidés qui harcèlent les troupeaux.

\section{2) Dans le canton Assalé (Tchad)}

- Les prélèvements ont été étendus à d'autres espèces que les bovins : chèvres, chevaux, ânes et dromadaires. Les taux d'infestation chez les bovins peuvent être ici comparés au total des animaux composant les troupeaux et au total de ceux examinés.

- Les résultats par espèces sont les suivants :

ZABISEAL $\because$ I

\begin{tabular}{|c|c|c|c|c|c|}
\hline & Bovins & Shevalu & Irea & Chêvres & Dromadaires \\
\hline Lffectu1 tetal & 5456 & - & - & - & - \\
\hline $\begin{array}{l}\text { Nombre d'àiraux } \\
\text { exuninés }\end{array}$ & 253 & $\angle 1$ & $2=$ & 13 & 13 \\
\hline $\begin{array}{l}\text { nembre ci'anical. } \\
\text { losttifs }\end{array}$ & 12 & 5 & 0 & 0 & 7 \\
\hline $\begin{array}{l}\text { 1. } 100 \text { sur leg etanaux } \\
\text { exemines }\end{array}$ & 4,72 & 12,19 & C & 0 & 53,24 \\
\hline $\begin{array}{l}\text { p.100 sur le Lotal } \\
\text { ces minald }\end{array}$ & 0,22 & - & - & - & - \\
\hline
\end{tabular}

- Les trypanosomes en cause sont Trypanosoma vivax et $T$. evansi, répartis ainsi :

TABLEAU $N^{\circ}$ II

\begin{tabular}{|l|c|c|c|}
\hline & Bovins & Chevaux & Dromadaires \\
\hline T. vivax & 9 & - & - \\
T. evansi & 3 & 5 & 7 \\
\hline Total & & & 7 \\
\hline
\end{tabular}

- Chez les bovins, 4,74 p. 100 de l'ensemble des animaux examinés sont parasités. Les valeurs extrêmes observées dans certaines localités sont de 2,32 et 12,50 p. 100. Elles se révèlent très inférieures à celles enregistrées dans la région de Makari.

- Dans le canton Assalé, la région de Karal paraît constituer la limite des aires de répartition des trypanosomes à $T$. vivax et à $T$. evansi. Ces 2 espèces peuvent se rencontrer simultanément en 
raison de la réunion des troupeaux venus du Nord et du Sud. Les transmissions mécaniques sont assurées par l'intervention des insectes hématophages.

\section{c) Conclusion}

Mis à part les quelques foyers du sultanat de Makari qui révèlent un taux de parasitisme élevé, l'ensemble de la région où s'est déroulée la campagne de lutte contre les glossines n'apparaît pas devoir être considérée comme fortement infestée, bien que les sondages aient été faits après la saison des pluies, période la plus favorable aux transmissions mécaniques et à l'extension du parasitisme.

\section{Sondages de 1977}

- Plus de 2 ans après l'élimination des glossines dans la région du bas-Chari, les éleveurs du canton Assalé considérant que la trypanosomose sévissait encore dans leurs troupeaux malgré les traitements trypanocides pratiqués annuellement, il était donc nécessaire de préciser la cause des symptômes constatés, imputables, soit à une infestation massive à $T$. vivax ou $T$. evansi, rebelle aux traitements, soit à toute autre origine, parasitaire ou autre.

\section{a) Pratique des prélèvements}

- Les animaux examinés appartenaient à 3 troupeaux, 2 sédentaires et 1 transhumant:

- le $1^{\text {ex }}$ n'ayant pas reçu de trypanocides depuis plus de 6 mois;

- le $2^{\mathbf{e}}$ ayant été traité 2 mois plus tôt au Bérénil ;

- le $3^{e}$, appartenant à des nomades peuls, sort chaque année du canton pour effectuer de grands déplacements.

- Dans chaque troupeau, les prélèvements de sang ont été réalisés sur des animaux présentant des signes cliniques pouvant évoquer la trypanosomose : maigreur, adénites, larmoiement, kératite. Comme lors des sondages de 1974, il a été procédé à un examen de sang à l'état frais et à la confection de frottis, pour une cinquantaine d'animaux par troupeau.

- Des examens coprologiques, pratiqués sur les mêmes animaux du $1^{\text {er }}$ et du $3^{\text {e }}$ troupeau, ont complété les recherches de parasites sanguins.

\section{b) Résultats}

Aucun des bovins examinés dans chacun des troupeaux n'a été trouvé porteur de trypanosomes, malgré les signes cliniques présentés.

Dans le $1^{\text {er }}$ troupeau, ces signes pouvaient être rattachés à la fièvre aphteuse qui sévissait au moment des interventions. Mais des prélèvements de fèces sur les animaux les plus faibles ont révélé une infestation d'intensité moyenne en strongles et coccidies.

L'analyse coprologique de 23 prélèvements effectués sur des animaux malades du $3^{\circ}$ troupeau a montré que 22 d'entre eux étaient fortement parasités par les coccidies et 9 étaient porteurs de strongles. Schistosoma bovis et Paramphistomum sp. ont été rencontrés respectivement dans 4 et 5 analyses.

\section{c) Conclusion}

Les enquêtes faites en 1977 permettent de penser que les trypanosomoses bovines ont totalement disparu du canton Assalé à la suite de l'éradication des glossines et des traitements trypanocides appliqués pendant 2 ans. Mais le risque d'explosion de foyers localisés est toujours à craindre en raison de l'introduction possible d'animaux infectés et de la présence d'autres diptères hématophages.

La présence d'insectes piqueurs autres que les tsé-tsé, l'existence de troupeaux transhumants pouvant s'infecter ailleurs et le contact des bovins avec des dromadaires porteurs de $T$. evansi, sont des éléments susceptibles de créer des foyers localisés. L'attention vis-à-vis de ces maladies ne doit donc pas être relâchée.

Les symptômes cliniques attribués à la trypanosomose résultent de diagnostics erronés et sont probablement, selon ces enquêtes, imputables à un fort parasitisme intestinal négligé.

\section{DISCUSSION}

L'évolution des trypanosomoses animales après l'éradication locale et définitive des glossines n'a été jusqu'ici que très rarement étudiée.

Dans sa thèse de Doctorat Vétérinaire, C. Sauvel fait observer à ce sujet qu'en 1977, c'est-à-dire plus de 30 ans après la fin de la première campagne d'éradication par pulvérisation d'insecticides de contact, il n'existe dans la littérature professionnelle ou spécialisée qu'une seule étude 
(S. M. Touré, 1961) où ce problème soit objectivement exposé, ce qui l'amène à conclure qu'en la matière tout se passe comme si la disparition des glossines entraînait celle des trypanosomes concernés, de façon systématique et définitive.

Dans le cas qui fait l'objet de cette note, les sondages opérés montrent, en dépit de leur faible nombre, que dans la mesure où la disparition des glossines est complétée par une action trypanocide contre les parasites résiduels $\mathrm{du}$ bétail, ceux-ci disparaissent, en dépit de la présence d'une abondante faune locale d'insectes piqueurs aptes à transmettre mécaniquement les hémo-parasites en cause.

Ils montrent également que les suspicions, par les éleveurs, de maladies à trypanosomes ne sont pas toujours justifiées du fait de l'existence d'autres affections, parasitaires notamment, présentant des symptômes à peu près identiques.

Ce dernier point est d'une importance majeure car une insuffisance dans le diagnostic de la maladie en cause peut avoir des répercussions considérables tant en ce qui concerne la nécessité d'entreprendre une campagne d'éradication que dans l'appréciation de ses résultats.

La pathologie vétérinaire intertropicale accorde une place essentielle aux trypanosomoses. Sans mésestimer l'importance de ces affections, on peut remarquer que dans nombre de cas le rôle de premier plan qui leur est attribué n'est pas toujours appuyé sur des données particulièrement convaincantes. L'insuffisance des diagnostics et celle des traitements anti-parasitaires ne permettent pas de conclure à la prédominance de l'une ou l'autre des affections pouvant atteindre les animaux.

Pour les mêmes raisons le résultat de toute campagne contre ces maladies ne pourra être valablement apprécié que par l'étude systématique de tous les cas de morbidité présentant des symptômes évoquant les trypanosomoses.
La décision d'entreprendre une campagne d'éradication des glossines pour éliminer les trypanosomoses du bétail ne devrait donc être prise qu'à la suite d'enquêtes épidémiologiques complètes, sous peine d'aller parfois au devant de graves mécomptes tant pathologiques qu'économiques et ses résultats appréciés qu'après étude systématique de tous les cas de morbidité présentant des symptômes généraux pouvant faire suspecter la trypanosomose.

En conséquence, il paraît nécessaire de tecommander aux responsables de la santé animale dans les régions où sévissent les trypanosomoses animales d'établir en premier lieu la hiérarchie des interventions d'ordre sanitaire et médical à opérer compte tenu de celle des affections en cause.

\section{CONCLUSION GÉNÉRALE}

Les examens hématologiques et coprologiques effectués dans le canton Assalé à la fin de la campagne contre les glossines (1974) et 3 ans plus tard (1977) ont montré l'absence d'animaux trypanosomés, mais révélé un fort parasitisme intestinal. Quelques cas de strongylose et de nombreux cas de coccidiose ont été mis en évidence. Le mauvais état d'entretien et la morbidité observés chez certains animaux ne sont donc pas imputables à la trypanosomose comme le pensaient les éleveurs, mais à ces infestations parasitaires.

Ces quelques sondages pratiqués au Tchad abordent l'important problème des conséquences sanitaires de l'élimination des glossines dans une région préalablement infestée. Ils montrent l'intérêt qu'il faut porter aux relations entre les campagnes contre les tsé-tsé et la disparition des trypanosomoses pour être en mesure de répondre objectivement dans tous les cas à cette importante question : "l'éradication des glossines suffit-elle pour faire disparaître les trypanosomoses?

\section{SUMMARY}

Glossina and animal trypanosomiasis eradication : results of sample surveys in the lake Chad area

Sample surveys have been made in the Low-Chari Valley (Chad) in order to evaluate the after effects of glossina eradication and of mass trypanocide treatments on local trypanosoma epidemiology ( $T$. vivax and $T$. evansi) They reveal that two years after the end of the insecticide sprayings, breeders still attribute their herd mortality and morbidity to these hemo-parasites. The 
causes, in fact, were found to be a severe intestinal parasitism whose general symptoms could be confused with various parasitoses.

The authors acknowledge the effectiveness of the eradication methods used on local traypanosoma but they point out the necessity to carry out extensive epidemiological surveys prior to any glossina eradication campaign in order to establish a classification of local animal diseases according to their incidence as well as to determine means to fight them.

\section{RESUMEN \\ Eliminación de las glosinas y tripanosomiasis animal : Resultados de algunas encuestas en la region del lago Chad}

Se efectuaron encuestas en el valle del Bajo Chari en Chad para determinar la incidencia de la desaparición de las glosinas y de los tratamientos tripanocidos sobre la epidemiologia de las tripanosomiasis locales ( $T$. vivax y $T$. evansi). Muestran que dos años después el fin de las pulverizaciones, el parasitismo intestinal únicamente es causa de la mortalidad y de la morbidez de los animales aunque los ganaderos les atribuyen a dichos hemoparásitos. Se puede confundir los síntomas generales del parasitismo intestinal con los de otras enfermedades parasitarias. Según los autores, las medidas aplicadas para eliminar las tripanosomiasis locales son eficaces ; Sin embargo, se necesitan encuestas epidemiologicas completas, antes la realización de un programa de eradicación de glosinas para establecer el orden de importancia de las enfermedades locales y definir el de las acciones de lucha.

\section{BIBLIOGRAPHIE}

1. GRUVEL (J.). Contribution à l'étude écologique de Glossina tachinoides Westwood, 1850 (Diptera, Muscidae) dans la réserve de Kalamaloue, vallée du BasChari, Thèse Doct. Sci. nat., Paris. VI, 1972.

2. GRUVEL (J.). Contrôle de l'assainissement glossinaire dans les zones du projet Assalé-Serbewel. Rapport final ; N'Djaména, Laboratoire de recherches vétérinaires et zootechniques de Farcha, I. E. M. V. T., 1976.

3. GRUVEL (J.). Enquêtes sur la trypanosomose du bétail dans le canton Assalé-Serbewel. In : Rapport annuel du Laboratoire de Farcha (I. E. M. V. T. : région de recherches vétérinaires et zootechniques d'Afrique centrale), 1974, p. E39-E42.

4. GRUVEL (J.). Les trypanosomoses animales du Tchad : situation actuelle. Colloque O.I.E. sur les moyens de lutte contre les trypanosomoses et leurs vecteurs. Paris, 12-15 mars 1974. Ma1sons-Alfort, I. E. M. V. T., p. 301-302.

5. SAUVEL (C.). Glossines et insecticides. Thèse Doct. vét., Alfort, 1977, no 48.

6. TIBAYRENC (R.). La campagne de lutte contre les glossines dans le bassin du lac Tchad. I. Prospections, pulvérisations, premières conclusions. Rev. Elev. Méd. vét. Pays trop., 1977, 30 (1) : 19-30.

7. TIBAYRENC (R.), GRUVEL (J.). La campagne de lutte contre les glossines dans le bassin du lac Tchad. II. Contrôle de l'assainissement glossinaire. Critique technique et financière de l'ensemble de la campagne. Conclusions générales. Rev. Elev. Méd. vét. Pays trop., 1977, 30 (1) : 31-39. 